\title{
Multidimensional Comparison of Cancer-Related Fatigue and Chronic Fatigue Syndrome: The Role of Psychophysiological Markers
}

\author{
Hye Youn Park ${ }^{1}$, Hong Jun Jeon², Young Rong Bang ${ }^{3}$, and In-Young Yoon ${ }^{1,4} \bowtie$ \\ 'Department of Psychiatry, Seoul National University Bundang Hospital, Seongnam, Republic of Korea \\ ${ }^{2}$ Department of Psychiatry, Konkuk University Medical Center, Seoul, Republic of Korea \\ ${ }^{3}$ Department of Psychiatry, Dong-A University Hospital, Busan, Republic of Korea \\ ${ }^{4}$ Department of Psychiatry, Seoul National University College of Medicine, Seoul, Republic of Korea
}

\begin{abstract}
Objective The present study compared cancer-related fatigue (CRF) and chronic fatigue syndrome (CFS) using multidimensional measurements with the aim of better understanding characteristics and exploring markers of two similar fatigue syndromes.

Methods Twenty-five patients with CRF and twenty patients with CFS completed questionnaires, including the Fatigue Severity Scale (FSS), Hospital Anxiety Depression Scale (HADS), Perceived Stress Scale (PSS), and Pittsburgh Sleep Quality Index (PSQI). Additionally, levels of high sensitivity C-reactive protein (hs-CRP), heart rate variability (HRV), and electroencephalography (EEG) were obtained. Neurocognitive functioning was also evaluated.

Results Both groups showed comparable levels of psychological variables, including fatigue. Compared to CFS subjects, CRF patients had significantly higher hs-CRP levels and a reduced HRV-index. The within-group analyses revealed that the FSS score of the CRF group was significantly related to scores on the HADS-anxiety, HADS-depression, and PSQI scales. In the CFS group, FSS scores were significantly associated with scores on the PSS and the absolute delta, theta, and alpha powers in frontal EEG.

Conclusion Findings indicate that different pathophysiological mechanisms underlie CFS and CRF. Inflammatory marker and HRV may be potential biomarkers for distinguishing two fatigue syndromes and frontal EEG parameters may be quantitative biomarkers for CFS.

Psychiatry Investig 2019;16(1):71-79
\end{abstract}

Key Words Fatigue, Cancer, Biomarkers, Electrocardiography, Electroencephalography.

\section{INTRODUCTION}

There is a high prevalence of cancer-related fatigue (CRF), which is prolonged fatigue after treatment for cancer, in this population. ${ }^{1}$ However, the pathophysiological mechanisms underlying CRF are not yet clearly understood. Most criteria for CRF consist of core characteristics that include complaints of fatigue that result in functional impairments accompanied by cognitive and sleep disturbances. ${ }^{2,3}$ These components

Received: October 19, 2017 Revised: June 9, 2018

Accepted: October 26, 2018

$\triangle$ Correspondence: In-Young Yoon, MD, PhD

Department of Psychiatry, Seoul National University Bundang Hospital, 82 Gumi-ro 173beon-gil, Bundang-gu, Seongnam 13620, Republic of Korea Tel: +82-31-787-7433, Fax: +82-31-787-4058, E-mail: iyoon@snu.ac.kr

(a) This is an Open Access article distributed under the terms of the Creative Commons Attribution Non-Commercial License (https://creativecommons.org/licenses/bync/4.0) which permits unrestricted non-commercial use, distribution, and reproduction in any medium, provided the original work is properly cited. overlap with the diagnostic criteria for chronic fatigue syndrome (CFS), ${ }^{4}$ which is characterized by intense persisting fatigue that limits function. Thus, it has been proposed that similar mechanisms, including inflammation and perpetual deconditioning, underlie these two fatigue syndromes. ${ }^{5}$ Still, the pathophysiology of CFS remains elusive, immune and neuroendocrine dysfunction and psychiatric disorders may be related to this disorder. ${ }^{6}$ Additionally, central nervous system (CNS) abnormalities appear to be involved in the pathophysiology of CFS however, the results have been inconclusive. ${ }^{4}$

Despite the similarities of these two fatigue syndromes, few studies have specifically compared them. Servaes et al. ${ }^{5}$ reported that CFS patient exhibit a more severe degree of fatigue and pain than patients with CRF. Previous study reported the associations between cognitive function and the $\mathrm{CRF}^{7}$ and CFS. ${ }^{8}$ However there has been very little comparative examination of cognitive dysfunctions between two syn- 
dromes using objective cognitive tests. Recently, Light et al. ${ }^{9}$ identified differential gene expressions related to immune and energy pathways between patients with CRF and CFS but no comparative studies have employed psychophysiological measures such as electroencephalography (EEG) or heart rate variability (HRV), which represent reproducible parameters that can complement psychosomatic performance measures. Resting-state quantitative EEG (qEEG) measures serve as an indicator of brain function and offer a noninvasive assessment of mental aspects of fatigue which is associated with cognitive fatigue. ${ }^{10}$ While, HRV is an objective measure of autonomic nervous system (ANS) regulation that reflects stress. ${ }^{11}$ Recent studies have reported lower values for HRV parameters in cancer patients and comparable values in CFS patients compared to healthy controls; these studies concluded that these changes are specifically related to fatigue. ${ }^{12-14}$ Previous research using qEEG measures have found unstable EEGvigilance in CRF patients ${ }^{15}$ and altered delta and beta activities in $\mathrm{CFS}^{16,17}$ patients compared to healthy controls.

These two distinct illnesses may be result from using different strategies to adapt to somatic conditions with different etiologies. A biomarker is defined as an indicator of a biologically derived pathogenic process, and the identification of distinct biomarkers for these two fatigue syndromes may improve our understanding of the biology of fatigue. Furthermore, biomarkers may aid in the differential diagnosis and proper management of patients with chronic, uniform fatigue, especially in oncology settings. The present comparative study examined the shared and specific factors associated with CRF and CFS. For this comparison, multifactorial assessments of chronic fatigue were performed. Additionally, this exploratory study aimed to identify potential objective biomarkers for each fatigue syndrome.

\section{METHODS}

\section{Subjects}

Subjects were recruited from Seoul National University Bundang Hospital (SNUBH) between November 2013 and September 2015. A total of 68 subjects were screened and 48 provided consent to participate. Of those who provided consent, 45 ( 25 cancer patients and 20 controls) were eligible and enrolled in this study.

The CRF group consisted of patients with non-metastatic cancer who were experiencing significant fatigue after primary treatment for cancer. All participants were recruited from outpatients of cancer center in the SNUBH. The diagnostic criteria for CRF used in the present study were introduced by previous studies ${ }^{18}$ as follows: 1) the presence of fatigue for at least 2 weeks and at least 5 fatigue-related symptoms,
2) the significant impact on work or self-care, 3) the fatigue symptoms are caused by cancer or cancer therapy, 4) the fatigue symptoms are not primarily caused by co-morbid psychiatric disorder. Exclusion criteria were 1) history of a major psychiatric disorder, 2) history of a cardiovascular disorder or event, 2) ongoing treatment with chemotherapy or radiotherapy, and 3) suicidal risk. The subjects in the CFS group were enrolled from outpatients attending fatigue clinic or psychiatric clinic in the SNUBH based on the Oxford criteria for $\mathrm{CFS}^{19}$ as follows: 1) the presence of fatigue as a main symptom, 2) the significant disability, 3) the absence of a medical or psychiatric disease plausible for fatigue. Subjects with a cardiovascular disorder or event and concurrent medication that may impact on the results were excluded.

The diagnostic interviews of both the CRF and CFS subjects were conducted by three psychiatrists who applied the Diagnostic and Statistical Manual of Mental Disorders, Fourth Edition (DSM-IV) criteria and used the Structured Clinical Interview for DSM-IV (SCID) $)^{20}$ to exclude major psychiatric diagnoses.

All subjects included in this study were diagnosed with moderate to severe fatigue, which was diagnosed based on a cut-off score $\geq 4$ on the Brief Fatigue Inventory (BFI). ${ }^{21}$ Informed consent was obtained from all subjects after the study information was offered and the study protocol was approved by the Institutional Review Board of SNUBH (No. B-1308/213-003).

\section{Psychological measurements}

The BFI includes nine items that are scored on 10-point Likert scales. The Cronbach's a was 0.88 in the present sample.

The Fatigue Severity Scale (FSS) was developed to assess fatigue in patients with chronic diseases. ${ }^{22}$ The Cronbach's $\alpha$ was 0.92 .

The Korean version of the Hospital Anxiety and Depression Scale (K-HADS) was used to evaluate emotional stress in the study subjects. ${ }^{23}$ The Cronbach's $\alpha$ in this study was 0.84 .

The Perceived Stress Scale (PSS) evaluates one's level of perceived stress. ${ }^{24}$ The Cronbach's a was 0.77 .

Subjective quality and quantity of sleep were assessed with the Pittsburgh Sleep Quality Index (PSQI). ${ }^{25}$ The Cronbach's a was 0.81 .

The Functional Assessment of Cancer Therapy General (FACTG) was used to evaluate quality of life (QoL) in the patients with cancer (CRF group). ${ }^{26}$ This scale is comprised of the following four domains: physical well-being, social/family well-being, emotional well-being, and functional well-being. The internal consistency was $\alpha=0.79$ in the present study.

\section{Laboratory test for systemic inflammation}

Blood samples were drawn to determine serum levels of 
high sensitivity C-reactive protein (hs-CRP); all samples were measured with a high-sensitive particle-enhanced immunoturbidimetric assay.

\section{Physiological measurements}

For the HRV analyses, high-resolution $(1,000 \mathrm{~Hz})$ electrocardiograms (ECGs) were collected over 8 min using the Synamps 2 amplifier (Compumedics; Melbourne, Australia). The ECG was recorded from two electrodes attached to both supraclavicular areas. All analyses were performed using HRV analysis software (Telescan version 2.0, Laxtha, Daejeon, Korea). The following parameters were extracted for the time domain: mean heart period (HP), standard deviation of the $\mathrm{NN}$ (SDNN), proportion of NN50 (PNN50), root mean square of the differences in successive NNs (RMSSD), and HRV index. Parametric auto-regression was processed on the power spectral domain analysis and the power levels of the low-frequency (LF; 0.04-0.15 Hz) and high-frequency (HF; 0.15-0.4 $\mathrm{Hz}$ ) components in the absolute and normalized units (divided by $\mathrm{LF}+\mathrm{HF}$ ) were determined.

To examine EEG characteristics related to mental fatigue, EEG data were acquired immediately after completing 1 hour of neurocognitive testing. The EEG signals were recorded from the scalp with a 64-channel EEG acquisition system over 15 min while the subjects were sitting with their eyes closed. Electrodes were placed according to the extended international 10-20 system. The EEG signals were amplified and digitalized with a 64-channel Neuroscan Synamps (Compumedics USA) with a sampling rate of $1 \mathrm{kHz}$. A frequency analysis was performed in the frequency domain to investigate fatigue-related CNS signal differences and a Fast Fourier Transform (FTT) was applied to selected 120-s segments without eye blinks or other artifacts. The absolute values of EEG power were extracted for five bands (delta: $1.0-4.0 \mathrm{~Hz}$, theta: 4.0-8.0 Hz, alpha: $8.0-12.0 \mathrm{~Hz}$, beta: $12.0-25.0 \mathrm{~Hz}$, and high beta: $25.0-30.0 \mathrm{~Hz}$ ) and the absolute power of each electrode was averaged into five grouped cerebral regions: frontal, central, parietal, temporal, and occipital. The power values from the electrodes on both hemispheres were averaged because the hemispheric asymmetry analyses were not significant.

\section{Neurocognitive tests}

Three neurocognitive tests were administered to assess attention, executive function, verbal learning, and working memory in relation to CRF and $\mathrm{CFS}^{27}$ : the Trail Making Test (TMT), Continuous Performance Test (CPT), and Auditory Verbal Learning Test (AVLT). All tests were performed using the Computerized Neurocognitive Function Test (CNT4.0, Maxmedica Inc., Seoul, Korea). ${ }^{28}$

\section{Statistical analysis}

The demographic and clinical characteristics and categorical variables were analyzed with either Pearson's chi-squared $\left(\chi^{2}\right)$ tests or Fisher's exact tests, while t-tests or Mann-Whitney $\mathrm{U}$ tests were used to analyze the continuous variables. Absolute power and mean power were log-transformed to normalize the data distributions. Comparisons of absolute power between the groups were performed with a two-way analysis of covariance (ANCOVA) adjusted for covariates was performed to compare the HRV and EEG variables. Withingroup analyses were conducted to evaluate the correlations between fatigue severity and the psychophysiological variables in each group. Two-tailed Pearson's correlation or Spearman's rho correlation coefficients (nonparametric test on hs-CRP) were used according to the results of normality tests using the Kolmogorov-Smirnov and Shapiro-Wilk tests. Additionally, univariate linear regression analyses were performed and these analyses were not adjusted for multiple testing thus $\mathrm{p}$ value $<0.05$ was considered to indicate statistical significance. All analyses were conducted using IBM SPSS Statistics ver. 20 (IBM Corp., Armonk, NY, USA).

To date, no studies have compared the EEG data of CRF and CFS subjects and, thus, the present study determined the effect size and calculated the appropriate sample size from a previous pilot study that investigated the EEG characteristics of CRF patients. 15 The required total sample size was $\geq 38$ to obtain a power of 0.8 and an alpha value of 0.05 with two-tailed significance.

\section{RESULTS}

\section{Between-group analyses}

The demographic and clinical characteristics of both groups are presented in Table 1. There were no differences between the groups in terms of fatigue severity, level of anxiety, depression stress, or sleep disturbances. Compared to the CFS group, the CRF group had a higher level of hs-CRP; this difference remained statistically significant after adjusting for age. The comparisons of the HRV parameters revealed that the CRF group had significantly lower PNN50 (\%) and HRV index values than the CFS group. Additionally, in the frequency domain, LF power was significantly lower in the CRF group than the CFS group after adjusting for age. A recent study produced Korean norms for HRV parameters, and we included these normative data as reference values for controls (Table 2). ${ }^{29}$

In the neurocognitive tests, the mean time to complete the TMT-B was longer in the CRF group than in the CFS group but there was no difference between the groups after adjustment with covariates (age and educational level) and Bonferroni correction for multiple comparisons. Spectral analyses of 
absolute EEG powers and two-way ANCOVA tests were performed for each cortical region but there was no significant difference (Table 3).

\section{Within-group analyses}

The partial correlation analysis and univariate linear regression analysis examined the relationships between fatigue severity (FSS score) and the psychophysiological variables,

Table 1. Demographic and clinical characteristics of the subjects

\begin{tabular}{|c|c|c|c|c|}
\hline Variables & CRF group $(\mathrm{N}=25)$ & CFS group $(\mathrm{N}=20)$ & t-value & p-value \\
\hline Age (years)* & $55.52 \pm 9.39$ & $50.05 \pm 9.25$ & 1.953 & 0.057 \\
\hline Sex $(\text { male/female })^{\dagger}$ & $9 / 16$ & $7 / 13$ & 0.005 & 0.944 \\
\hline Educational level $^{\dagger}$ & & & 4.346 & 0.226 \\
\hline Elementary school & $0(0)$ & $1(5)$ & & \\
\hline Middle school & $3(12)$ & $0(0)$ & & \\
\hline High school & $12(48)$ & $8(40)$ & & \\
\hline$\geq$ University & $10(40)$ & $11(55)$ & & \\
\hline \multicolumn{5}{|l|}{ Type of cancer ${ }^{\dagger}$} \\
\hline Breast & $10(40)$ & & & \\
\hline Gastrointestinal & $6(24)$ & & & \\
\hline Lung & $5(20)$ & & & \\
\hline Thyroid & $4(16)$ & & & \\
\hline \multicolumn{5}{|c|}{ Time since the treatment finished ${ }^{\dagger}$} \\
\hline$<2$ years & $12(48)$ & & & \\
\hline$\geq 2$ years & $13(52)$ & & & \\
\hline
\end{tabular}

${ }^{*}$ data given as mean \pm standard deviation, ${ }^{\dagger}$ data given as number (\%). CRF: cancer-related fatigue, CFS: chronic fatigue syndrome

Table 2. Psychophysiological variables and inflammatory marker

\begin{tabular}{|c|c|c|c|c|c|}
\hline Variables & CRF group $(\mathrm{N}=25)$ & CFS group $(\mathrm{N}=20)$ & & t-value/z score & p-value \\
\hline \multicolumn{6}{|l|}{ Self-questionnaires } \\
\hline BFI & $6.26 \pm 1.41$ & $6.87 \pm 1.57$ & & -1.37 & 0.179 \\
\hline FSS & $6.66 \pm 1.21$ & $6.89 \pm 1.36$ & & -0.60 & 0.551 \\
\hline HADS-anxiety & $8.64 \pm 3.24$ & $10.50 \pm 4.41$ & & -1.63 & 0.110 \\
\hline HADS-depression & $10.08 \pm 2.63$ & $11.05 \pm 3.38$ & & -1.084 & 0.285 \\
\hline PSS & $20.36 \pm 4.91$ & $22.35 \pm 5.34$ & & -1.30 & 0.201 \\
\hline PSQI & $10.44 \pm 4.05$ & $9.35 \pm 3.59$ & & 0.94 & 0.351 \\
\hline \multicolumn{6}{|l|}{ Inflammatory marker } \\
\hline hs-CRP (mg/dL)* & $0.35 \pm 0.57$ & $0.08 \pm 0.14$ & & -2.403 & 0.016 \\
\hline Heart rate variability & & & (Reference value) & & \\
\hline Mean HP (ms) & $938.55 \pm 132.8$ & $916.19 \pm 111.63$ & - & 0.59 & 0.557 \\
\hline SDNN (ms) & $37.10 \pm 18.27$ & $46.20 \pm 16.65$ & $(41 \pm 18)$ & -1.70 & 0.097 \\
\hline PNN50 (\%) & $28.61 \pm 18.49$ & $39.98 \pm 12.97$ & - & -2.36 & 0.023 \\
\hline RMSSD (ms) & $33.11 \pm 28.20$ & $35.74 \pm 26.68$ & $(24 \pm 15)$ & -0.33 & 0.748 \\
\hline HRV index & $10.60 \pm 3.71$ & $13.40 \pm 4.25$ & - & -2.31 & 0.026 \\
\hline $\mathrm{LF}\left[\ln \left(\mathrm{ms}^{2}\right)\right]$ & $4.98 \pm 0.96$ & $5.59 \pm 0.71$ & $(5.70 \pm 1.05)$ & -2.40 & 0.021 \\
\hline Normalized LF (nu) & $48.36 \pm 16.23$ & $55.06 \pm 16.02$ & $(67.9 \pm 18.8)$ & -1.36 & 0.182 \\
\hline $\mathrm{HF}\left[\ln \left(m s^{2}\right)\right]$ & $5.06 \pm 0.89$ & $5.37 \pm 0.89$ & $(5.27 \pm 1.14)$ & -1.14 & 0.260 \\
\hline Normalized HF (nu) & $51.64 \pm 16.23$ & $44.94 \pm 16.02$ & $(32.1 \pm 18.8)$ & 1.36 & 0.182 \\
\hline
\end{tabular}

Data given as mean \pm standard deviation. ${ }^{*}$ Mann-Whitney U test. Reference values are based on the normative HRV parameters in Koreans. ${ }^{29}$ CRF: cancer-related fatigue, CFS: chronic fatigue syndrome, BFI: Brief Fatigue Inventory, FSS: Fatigue Severity Score, HADS: Hospital Anxiety and Depression Scale, PSS: Perceived Stress Scale, PSQI: Pittsburgh Sleep Quality Index, hs-CRP: high sensitivity C-reactive protein, HP: heart period, SDNN: standard deviation of normal beat to beat intervals, PNN: proportion of NN50, RMSSD: root mean square successive difference, LF: low frequency power, HF: high frequency power 
including the psychological questionnaires, hs-CRP levels, HRV parameters, and qEEG data after adjusting for age. In the CRF group, the FSS score was significantly related to the psychological variables [HADS-anxiety: $r=0.500$, regression coefficient $(b)=0.187, p=0.011$; HADS-depression: $r=0.451$, $\mathrm{b}=0.208, \mathrm{p}=0.024$; and PSQI: $\mathrm{r}=0.530, \mathrm{~b}=0.154, \mathrm{p}=0.008$ ] (Figure 1). The psychological variables related to the QoL dimensions (FACTG subscale) were also assessed and revealed that the FSS score was negatively correlated with the physical well-being subscale $(\mathrm{r}=-0.544, \mathrm{~b}=-3.189, \mathrm{p}<0.001)$ after controlling for the other psychological variables.

In the CFS group, the FSS score was correlated with the PSS score $(r=0.547, b=0.162, p=0.013)$, RMSSD ( $r=-0.509, b=$ -0.032, $\mathrm{p}=0.026)$, LF power $(\mathrm{r}=0.484, \mathrm{~b}=0.047, \mathrm{p}=0.036)$, and HF power $(r=-0.508, b=-0.051, p=0.026)$. The FSS score was also correlated with the absolute delta $(r=0.487, b=3.982$,

Table 3. Comparisons of neurocognitive performances and absolute electroencephalography (EEG) powers in frontal region

\begin{tabular}{|c|c|c|c|c|}
\hline Variables & CRF group $(\mathrm{N}=25)$ & CFS group $(\mathrm{N}=20)$ & $\mathrm{t}$-value & $\mathrm{p}$-value \\
\hline \multicolumn{5}{|l|}{ Neurocognitive tests } \\
\hline ACPT-omission $(\mathrm{N})$ & $7.13 \pm 8.64$ & $4.90 \pm 4.69$ & 1.03 & 0.308 \\
\hline ACPT-comission $(\mathrm{N})$ & $4.29 \pm 6.64$ & $3.00 \pm 2.47$ & 0.82 & 0.416 \\
\hline ACPT-response time (msec) & $0.85 \pm 0.09$ & $0.83 \pm 0.07$ & 0.61 & 0.545 \\
\hline RAVLT-Trial A1 (N) & $5.61 \pm 1.53$ & $6.15 \pm 1.50$ & -1.18 & 0.245 \\
\hline RAVLT-Trial A5 (N) & $10.65 \pm 3.46$ & $12.20 \pm 2.24$ & -1.71 & 0.094 \\
\hline Delayed free recall $(\mathrm{N})$ & $9.57 \pm 3.59$ & $10.20 \pm 2.69$ & -6.48 & 0.521 \\
\hline Delayed cued recall $(\mathrm{N})$ & $11.65 \pm 4.92$ & $13.65 \pm 1.57$ & -1.84 & 0.076 \\
\hline TMT-A-time (sec) & $35.24 \pm 18.90$ & $28.55 \pm 8.52$ & 1.47 & 0.150 \\
\hline TMT-A-error (N) & $2.28 \pm 3.09$ & $1.90 \pm 2.73$ & 0.43 & 0.668 \\
\hline TMT-B-time (sec) & $64.80 \pm 30.41$ & $48.05 \pm 16.65$ & 2.35 & 0.024 \\
\hline TMT-B-error (N) & $2.68 \pm 2.75$ & $1.55 \pm 1.67$ & 1.61 & 0.114 \\
\hline \multicolumn{5}{|l|}{ Absolute EEG powers (log) } \\
\hline Frontal delta power & $1.21 \pm 0.61$ & $1.24 \pm 0.18$ & -0.44 & 0.661 \\
\hline Frontal theta power & $0.98 \pm 0.24$ & $1.00 \pm 0.30$ & -0.26 & 0.795 \\
\hline Frontal alpha power & $1.02 \pm 0.39$ & $0.96 \pm 0.46$ & 0.51 & 0.611 \\
\hline Frontal beta power & $1.00 \pm 0.28$ & $0.98 \pm 0.25$ & 0.31 & 0.755 \\
\hline Frontal high beta power & $0.32 \pm 0.28$ & $0.29 \pm 0.26$ & 0.44 & 0.665 \\
\hline
\end{tabular}

Data given as mean \pm standard deviation. CRF: cancer-related fatigue, CFS: chronic fatigue syndrome, ACTP: Auditory Continuous Performance Test, RAVLT: Rey Auditory Verbal Learning Test, TMT: Trail Making Test
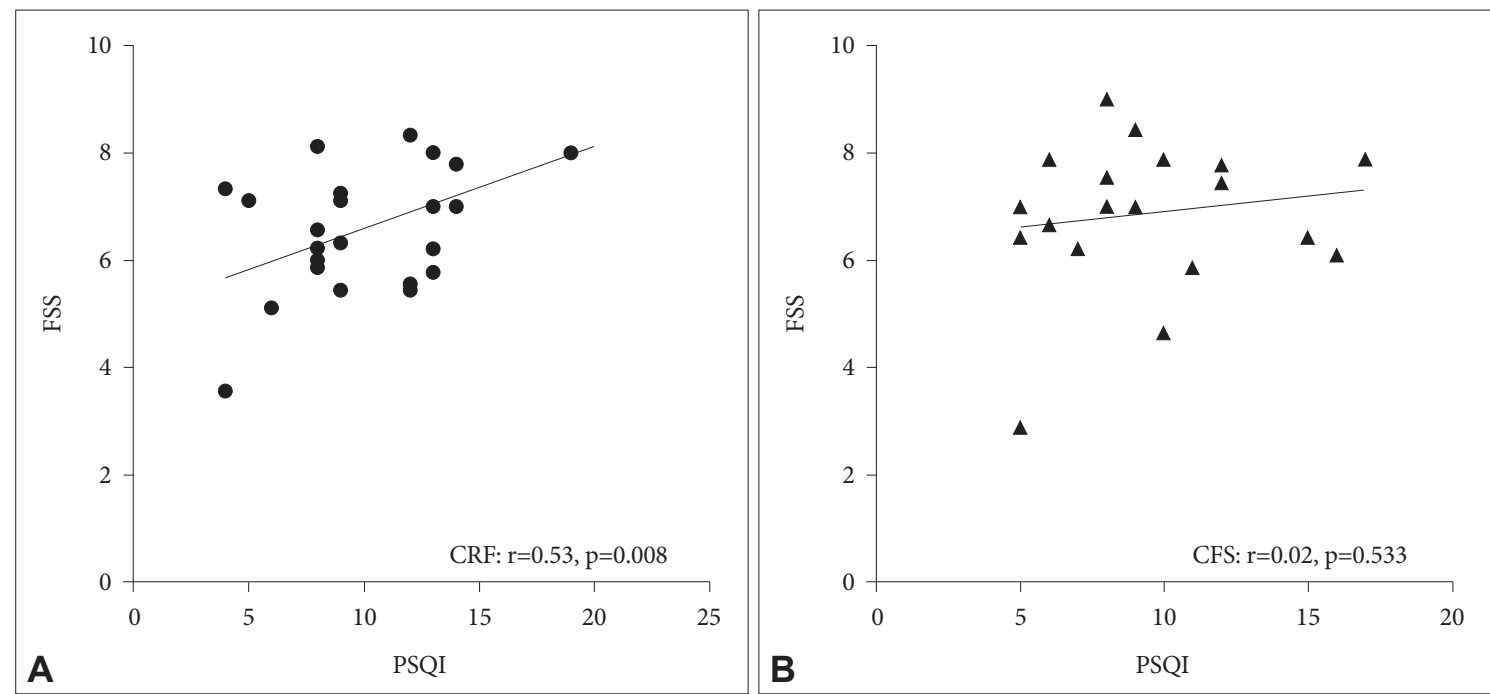

Figure 1. Plot of correlations between PSQI scores and FSS scores in the CRF (A) and CFS (B) groups. Lines show linear regression within each group. FSS: Fatigue Severity Score, PSQI: Pittsburgh Sleep Quality Index, CRF: cancer-related fatigue, CFS: chronic fatigue syndrome. 

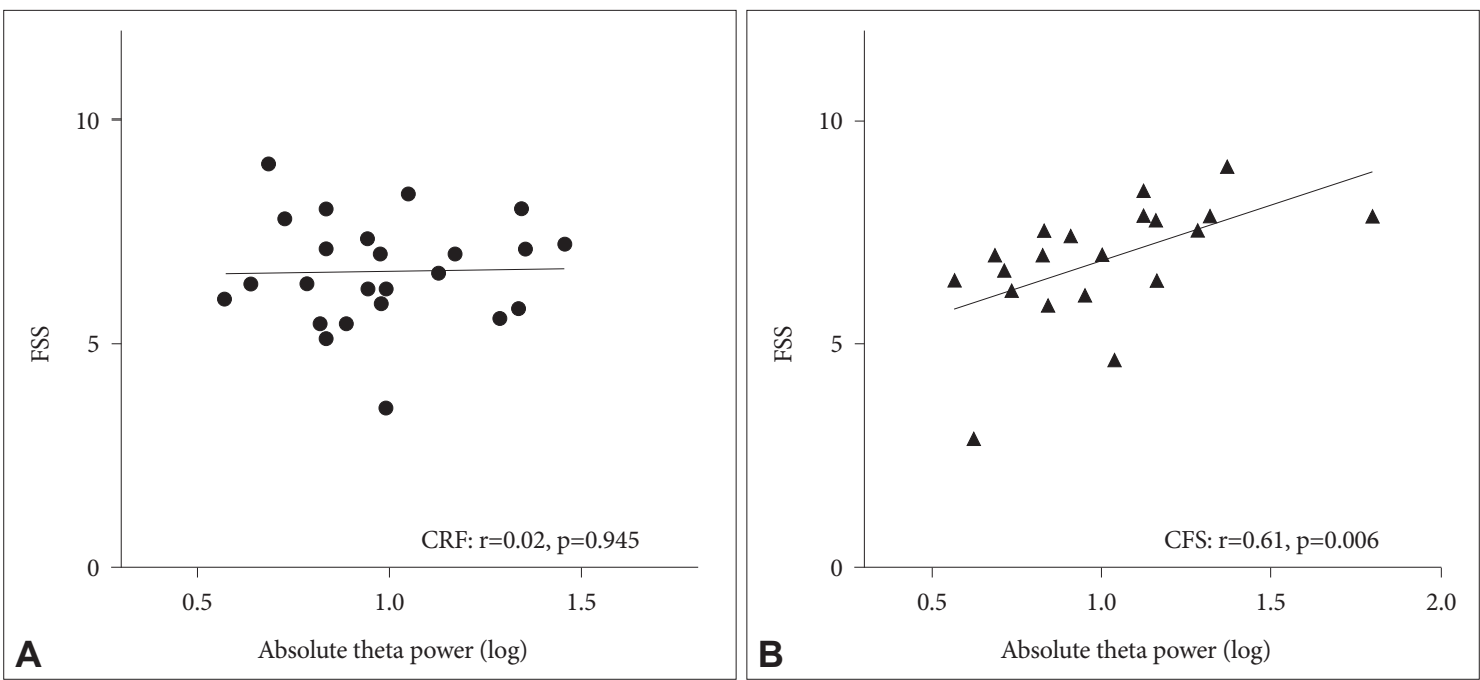

Figure 2. Plot of correlations between FSS scores and absolute theta EEG power in the frontal region in the CRF (A) and CFS (B) groups. Lines show linear regression within each group. FSS: Fatigue Severity Score, EEG: electroencephalogram, CRF: cancer-related fatigue, CFS: chronic fatigue syndrome.

$\mathrm{p}=0.034)$, theta $(\mathrm{r}=0.610, \mathrm{~b}=2.849, \mathrm{p}=0.006)$ (Figure 2$)$, and alpha $(\mathrm{r}=0.476, \mathrm{~b}=1.407, \mathrm{p}=0.039)$ powers in the frontal region.

\section{DISCUSSION}

The present study investigated the similarities and differences of CRF and CFS. In terms of the biological measurements, the CRF group had higher levels of hs-CRP and reduced values for PNN50, the HRV index, and LF power. The within-group analyses revealed that the severity of CRF was related to anxiety, depression, and sleep disturbances. On the other hand, the severity of CFS was associated with perceived stress, RMSSD, LF, and HF power from among the HRV parameters, and frontal delta, theta, and alpha activities from the qEEG variables.

In a previous qualitative study, Bennett et al. ${ }^{27}$ found that, except for pain, both CRF and CFS exhibited similar core symptoms of fatigue, cognitive deficits, and mood disturbances. Consistent with previous findings, the present study showed that patients with CRF were similar to patients with CFS in terms of psychological variables. Although we excluded potential subjects if an alternative major psychiatric disorder could explain their severe fatigue, it is readily apparent that depressive symptoms (measured by the HADS) and sleep disturbances (measured by the PSQI) were prevalent, similar to the previous study. ${ }^{27}$ The HADS used in the present study offers the advantage of reducing potential contamination with a measure of fatigue as it does not include the physical symptoms common to depression and fatigue. ${ }^{30}$ Some studies have suggested that, as fatigue and depression are independent conditions in cancer patients, depression is a comorbid condition of CRF. ${ }^{31}$ However, according to the litera- ture, depression and poor sleep are consistently associated with both fatigue syndromes due to common mechanisms, and they are also confirmed predisposing factors for the development of CRF as well as CFS. 4,30,32 The causality and directionality of these relationships remain uncertain; thus, further longitudinal studies are needed. In terms of inflammation, higher levels of hs-CRP were observed in the CRF group compared to the CFS group. The level of CRP has been consistently identified as a reliable biomarker of CRF but its relationship to CFS was inconclusive in a multiple adjusted analysis. ${ }^{33,34}$ Active inflammatory reactions caused by cancer or anticancer treatments might have influenced this group difference. ${ }^{34} \mathrm{Re}-$ cent studies have reported lower values for HRV parameters in cancer patients, which are specifically related to fatigue. ${ }^{12,13}$ Tumor-related factors, anticancer treatment, and distress modulate the ANS and alter HRV in patients with cancer. ${ }^{35}$ On the other hand, CFS patients show a similar pattern of HRV when compared with healthy controls; ${ }^{14}$ however, fewer studies have assessed HRV in CFS patients. Our results support previous findings showing that CFS patients presented higher SDNN, RMSSD and HF values than those of the control group, and obtained values intermediate between those of the CRF and control groups with regard to the LF, normalized LF, and normalized HF which implies that fatigue and cancer may be independently related to altered HRV parameters; however, this remains inconclusive due to the limitations of indirect comparisons. In the frequency domain of the HRV parameters, patients with CFS in the present study had relatively higher level of LF power, which represent sympathetic activity and tend to be elevated in individuals with mental stress. ${ }^{36}$ Thus, higher sympathetic tone in CFS patients may reflect a mental aspect of the CFS. The present findings sug- 
gest that hs-CRP and HRV variables may be biomarkers that reflect different mechanisms from other insults between two syndromes. However, the present study is the first to directly compare the hs-CRP, HRV variables of CRF and CFS patients and the present results should be interpreted with caution. Regarding neurocognitive functioning, previous studies using formal cognitive tests with CRF patients found impairments in attention and verbal memory. ${ }^{37}$ Similar results have been reported in patients with $\mathrm{CFS},{ }^{38}$ but there have been discrepancies in the prevalence and domains affected. This is the first comparison to measure the symptoms related to both fatigue syndromes using neurocognitive tests, and we observed no significant differences in the neurocognitive functioning of the two groups; this supports previous findings ${ }^{27}$ showing that cognitive impairments may be related to chronic fatigue rather than to a specific fatigue syndrome. Future studies with larger sample and measures of multiple cognitive domains could clarify these relationships.

Similar to previous studies, ${ }^{18,39}$ the present findings indicate that depression, anxiety, and insomnia are possible quantitative markers predictive of the severity of CRF in cancer patients, even though these factors are not specific to CRF. Additionally, we observed that CRF was associated with the physical well-being of cancer patients after adjusting for other psychological factors. Previous research reporting predictors that discriminated among the different dimensions (e.g., emotional, mental, and physical fatigue) of CRF demonstrated that depression was related to all types of fatigue and that high scores on the PSQI were associated with the severity of physical fatigue. ${ }^{32}$ These results may imply that the association between CRF and physical well-being was mediated by the quality of sleep. In terms of inflammation, higher levels of CRP are associated with greater fatigue in cancer patients. ${ }^{40}$ However, these associations were not observed in the present sample of CRF, this inconsistency may be due to our small sample size and other CRP-related factors, including body mass index (BMI), types of cancer, and medications, as a recent review pointed that these relationships were no longer significant after adjustment for medications and comorbidities. ${ }^{41}$ The EEG measures were not significantly related to fatigue severity in the CRF group in the present study. In a previous study, patients with cancer report persistent levels of physical fatigue while mental fatigue, as measured by EEG power, is prominent only during chemotherapy. ${ }^{10}$ These findings suggest that persistent CRF is more likely to be multidimensional fatigue beyond mental fatigue. ${ }^{42}$

In the CFS group, fatigue severity was associated with perceived stress. The relatedness between mental stress and CFS is mediated by dysfunctional ANS activity during the stress response. ${ }^{33}$ The present study observed negative associations between RMSSD and HF power and the severity of CFS. The RMSSD and HF power components are indices of parasympathetic activity. ${ }^{43}$ During mental stress, the ANS balance tends to shift toward parasympathetic withdrawal and it is widely accepted that decreased HF power is associated with psychological stress. ${ }^{44} \mathrm{~A}$ recent review found that patients with CFS exhibit reduced HRV and HF, but these changes were only significantly apparent during sleep..$^{14}$ Another study ${ }^{45}$ also reported correlations between the severity of CFS and HRV parameters. Though CFS patients showed relatively higher PNN50 (\%) and HRV index than those of CRF group in the present study, findings suggest that HRV measures may be non-invasive, quantitative markers that reflect the severity of CFS. This difference could be explained by the fact that patients with CFS are less likely to have other factors affecting HRV parameters such as medications and medical comorbidities. ${ }^{41}$

A variety of studies strongly suggest that CNS dysfunction is involved in CFS. ${ }^{4}$ Recent studies using qEEG measures ${ }^{16,17}$ have found altered delta activity in the fronto-limbic regions in CFS and suggested that delta activity could be a salient feature for differentiating between CFS and control subjects. ${ }^{46}$ While, Trejo et al. ${ }^{47}$ and Wascher et al. ${ }^{48}$ found that increased frontal theta power is associated with increased mental fatigue, because it might be assigned to the recruitment of executive function. In the present study, there were positive associations between the severity of CFS and slow wave (delta and theta) and alpha power activities in the frontal region. Taken together, these results regarding the relationships of slow and alpha waves with CFS severity provide evidence that mental fatigue is one component of a neurobiological mechanism underlying the pathophysiology of CFS.

There are several limitations should be considered. First, the statistical power of the present study may have been compromised by the modest sample size. Second, it was not possible to include healthy controls or cancer patients without chronic fatigue and this may have weakened the ability to distinguish between fatigue-related and cancer-related factors though we administered norm-referenced values. Finally, although age and educational level were adjusted for, it was not possible to control for medications, BMI, or individual activity, which could also affect physiological variables. ${ }^{41}$ Nonetheless, this is the first comparative study to assess CRF and CFS using physiological measures such as HRV and EEG parameters.

Two fatigue syndromes could have dissimilar mechanisms, such that inflammatory markers and HRV measures could be possible biomarkers used to differentiate these, especially in cancer patients or survivors. In the context of mental fatigue, frontal EEG parameters may contribute to quantitative biomarkers for CFS. Therefore, the present findings have po- 
tential implications that will contribute to a better understanding of two fatigue syndromes, these exploratory examinations of biomarkers that will aid in future researches on fatigue syndromes and their mechanisms.

\section{Acknowledgments}

The present study was funded by Seoul National University Bundang Hospital (Grant no. 02-2013-029).

\section{REFERENCES}

1. Stone P, Richards M, Hardy J. Fatigue in patients with cancer. Eur J Cancer 1998;34:1670-1676.

2. Cella D, Davis K, Breitbart W, Curt G; Fatigue Coalition. Cancer-related fatigue: prevalence of proposed diagnostic criteria in a United States sample of cancer survivors. J Clin Oncol 2001;19:3385-3391.

3. Piper BF, Cella D. Cancer-related fatigue: definitions and clinical subtypes. J Natl Compr Canc Netw 2010;8:958-966.

4. Afari N, Buchwald D. Chronic fatigue syndrome: a review. Am J Psychiatry 2003;160:221-236.

5. Servaes P, Prins J, Verhagen S, Bleijenberg G. Fatigue after breast cancer and in chronic fatigue syndrome: similarities and differences. J Psychosom Res 2002;52:453-459.

6. Avellaneda Fernandez A, Perez Martin A, Izquierdo Martinez M, Arruti Bustillo M, Barbado Hernandez FJ, et al. Chronic fatigue syndrome: aetiology, diagnosis and treatment. BMC Psychiatry 2009;9(Suppl 1):S1.

7. Stone PC, Minton O. Cancer-related fatigue. Eur J Cancer 2008;44:10971104.

8. Glass JM. Cognitive dysfunction in fibromyalgia and chronic fatigue syndrome: new trends and future directions. Curr Rheumatol Rep 2006; 8:425-429.

9. Light KC, Agarwal N, Iacob E, White AT, Kinney AY, VanHaitsma TA, et al. Differing leukocyte gene expression profiles associated with fatigue in patients with prostate cancer versus chronic fatigue syndrome. Psychoneuroendocrinology 2013;38:2983-2995.

10. Moore HC, Parsons MW, Yue GH, Rybicki LA, Siemionow W. Electroencephalogram power changes as a correlate of chemotherapy-associated fatigue and cognitive dysfunction. Support Care Cancer 2014;22: 2127-2131.

11. Vanderlei LCM, Pastre CM, Hoshi RA, Carvalho TDd, Godoy MFd. Basic notions of heart rate variability and its clinical applicability. Braz J Cardiovasc Surg 2009;24:205-217.

12. Crosswell AD, Lockwood KG, Ganz PA, Bower JE. Low heart rate variability and cancer-related fatigue in breast cancer survivors. Psychoneuroendocrinology 2014;45:58-66.

13. Vigo C, Gatzemeier W, Sala R, Malacarne M, Santoro A, Pagani M, et al. Evidence of altered autonomic cardiac regulation in breast cancer survivors. J Cancer Surviv 2015;9:699-706.

14. Meeus M, Goubert D, De Backer F, Struyf F, Hermans L, Coppieters I, et al. Heart rate variability in patients with fibromyalgia and patients with chronic fatigue syndrome: a systematic review. Semin Arthritis Rheum 2013;43:279-287.

15. Olbrich S, Sander C, Jahn I, Eplinius F, Claus S, Mergl R, et al. Unstable EEG-vigilance in patients with cancer-related fatigue (CRF) in comparison to healthy controls. World J Biol Psychiatry 2012;13:146-152.

16. Flor-Henry P, Lind JC, Koles ZJ. EEG source analysis of chronic fatigue syndrome. Psychiatry Res 2010;181:155-164.

17. Zinn MA, Zinn ML, Valencia I, Jason LA, Montoya JG. Cortical hypoactivation during resting EEG suggests central nervous system pathology in patients with Chronic Fatigue Syndrome. Biol Psychol 2018;136: 87-99.

18. Alexander S, Minton O, Andrews P, Stone P. A comparison of the characteristics of disease-free breast cancer survivors with or without can- cer-related fatigue syndrome. Eur J Cancer 2009;45:384-392.

19. Sharpe MC, Archard LC, Banatvala JE, Borysiewicz LK, Clare AW, David A, et al. A report--chronic fatigue syndrome: guidelines for research. J R Soc Med 1991;84:118-121.

20. Spitzer M, Robert L, Gibbon M, Williams J. Structured Clinical Interview for DSM-IV-TR Axis I Disorders, Research Version, Non-Patient Edition (SCID-I/NP). New York: Biometrics Research, New York State Psychiatric Institute; 2002.

21. Mendoza TR, Wang XS, Cleeland CS, Morrissey M, Johnson BA, Wendt JK, et al. The rapid assessment of fatigue severity in cancer patients: use of the Brief Fatigue Inventory. Cancer 1999;85:1186-1196.

22. Krupp LB, LaRocca NG, Muir-Nash J, Steinberg AD. The fatigue severity scale: application to patients with multiple sclerosis and systemic lupus erythematosus. Arch Neurol 1989;46:1121-1123.

23. Oh SM, Min KJ, Park DB. A study on the standardization of the hospital anxiety and depression scale for Koreans: a comparison of normal, depressed and anxious groups. J Korean Neuropsychiatr Assoc 1999;38: 289-296.

24. Cohen S, Williamson G. Perceived Stress in a Probability Sample of the US. In: Spacapam S, Editor. The Social Psychology of eHalth: Claremont Symposium on Applied Social Psychology. Newbury Park, CA: Sage, 1988, p 31-67.

25. Buysse DJ, Reynolds CF, Monk TH, Berman SR, Kupfer DJ. The Pittsburgh Sleep Quality Index: a new instrument for psychiatric practice and research. Psychiatry Res 1989;28:193-213.

26. Cella D. The Functional Assessment of Cancer Therapy-Lung and Lung Cancer Subscale assess quality of life and meaningful symptom improvement in lung cancer. Semin Oncol 2004;31:11-15.

27. Bennett B, Goldstein D, Friedlander M, Hickie I, Lloyd A. The experience of cancer-related fatigue and chronic fatigue syndrome: a qualitative and comparative study. J Pain Symptom Manage 2007;34:126-135.

28. . Kim SW, Shin IS, Kim JM, Yang SJ, Shin HY, Yoon JS. Association between attitude toward medication and neurocognitive function in schizophrenia. Clin Neuropharmacol 2006;29:197-205.

29. Lee CH, Lee JH, Son JW, Kim U, Park JS, Lee J, et al. Normative values of short-term heart rate variability parameters in Koreans and their clinical value for the prediction of mortality. Heart Lung Circ 2018;27: 576-587.

30. Brown LF, Kroenke K. Cancer-related fatigue and its associations with depression and anxiety: a systematic review. Psychosomatics 2009;50: 440-447.

31. Armes J, Krishnasamy M, Higginson I. Fatigue in Cancer. Oxford, UK: Oxford University Press; 2004.

32. Banthia R, Malcarne VL, Ko CM, Varni JW, Sadler GR. Fatigued breast cancer survivors: the role of sleep quality, depressed mood, stage and age. Psychol Health 2009;24:965-980.

33. Klimas NG, Broderick G, Fletcher MA. Biomarkers for chronic fatigue. Brain Behav Immun 2012;26:1202-1210.

34. Raison CL, Lin JM, Reeves WC. Association of peripheral inflammatory markers with chronic fatigue in a population-based sample. Brain Behav Immun 2009;23:327-337.

35. Arab C, Dias DPM, de Almeida Barbosa RT, de Carvalho TD, Valenti VE, Crocetta TB, et al. Heart rate variability measure in breast cancer patients and survivors: a systematic review. Psychoneuroendocrinology 2016;68:57-68.

36. Van Cauwenbergh D, Nijs J, Kos D, Weijnen L, Struyf F, Meeus M. Malfunctioning of the autonomic nervous system in patients with chronic fatigue syndrome: a systematic literature review. Eur J Clin invest 2014; 44:516-526.

37. Minton O, Stone PC. A comparison of cognitive function, sleep and activity levels in disease-free breast cancer patients with or without cancerrelated fatigue syndrome. BMJ Support Palliat Care 2012;2:231-238.

38. Michiels V, Cluydts R. Neuropsychological functioning in chronic fatigue syndrome: a review. Acta Psychiatr Scand 2001;103:84-93.

39. Bower JE. Cancer-related fatigue--mechanisms, risk factors, and treat- 
ments. Nat Rev Clin Oncol 2014;11:597-609.

40. Bower JE. Cancer-related fatigue: links with inflammation in cancer patients and survivors. Brain Behav Immun 2007;21:863-871.

41. Bower JE, Lamkin DM. Inflammation and cancer-related fatigue: mechanisms, contributing factors, and treatment implications. Brain, behavior, and immunity. Brain Behav Immun 2013;30(Suppl):S48-S57.

42. Wang Y, Shen J, Xu Y. Symptoms and quality of life of advanced cancer patients at home: a cross-sectional study in Shanghai, China. Support Care Cancer 2011;19:789-797.

43. Dogru MT, Aydin G, Tosun A, Keles I, Guneri M, Arslan A, et al. Correlations between autonomic dysfunction and circadian changes and arrhythmia prevalence in women with fibromyalgia syndrome. Anadolu Kardiyol Derg 2009;9:110-117.

44. Castaldo R, Melillo P, Bracale U, Caserta M, Triassi M, Pecchia L.
Acute mental stress assessment via short term HRV analysis in healthy adults: a systematic review with meta-analysis. Biomed Signal Process Control 2015;18:370-377.

45. Oh SJ, Ock SM, Yang MJ, Song CH, Ju SY. The correlations between phase angle of BIA, HRV and fatigue in chronic fatigue patients. Korean J Fam Pract 2015;5:163-167.

46. Jason LA, Zinn ML, Zinn MA. Myalgic Encephalomyelitis: Symptoms and Biomarkers. Curr Neuropharmacol 2015;13:701-734.

47. Trejo LJ, Kubitz K, Rosipal R, Kochavi RL, Montgomery LD. EEGbased estimation and classification of mental fatigue. Psychology 2015; 6:572-589.

48. Wascher E, Rasch B, Sanger J, Hoffmann S, Schneider D, Rinkenauer G, et al. Frontal theta activity reflects distinct aspects of mental fatigue. Biol Psychol 2014;96:57-65. 